Article

\title{
Impacto do ENOS na Variabilidade da Coluna Total de Ozônio Sobre a Região Nordeste do Brasil - Parte 2: La Niña Canônico e Modoki
}

\author{
Daniel Milano Costa de Lima ${ }^{1}$ (D), Mateus Dias Nunes ${ }^{2}$, Glauber Lopes Mariano ${ }^{1}$ \\ ${ }^{1}$ Curso de Mestrado em Meteorologia, Universidade Federal de Alagoas, Maceió, AL, Brasil. \\ ${ }^{2}$ Centro de Previsão de Tempo e Estudos Climáticos, Instituto Nacional de Pesquisas Espaciais, \\ Cachoeira Paulista, SP, Brasil.
}

Recebido em: 5 de Julho de 2020 - Aceito em: 25 de Novembro de 2020

\begin{abstract}
Resumo
Este estudo apresenta a segunda etapa da avaliação espaço-temporal das tendências a longo prazo nos valores da Coluna Total de Ozônio (CTO) sobre o Nordeste Brasileiro (NEB) durante a atuação do modo de variabilidade atmosférica El Niño - Oscilação Sul (ENOS), com foco na fase La Niña (modalidades Canônica e Modoki). O objetivo é analisar a variação anual e os efeitos desse fenômeno na região de estudo no período entre 1997 e 2018 . Os dados utilizados são provenientes de sensoriamento remoto, gerados pelos sensores Total Ozone Mapping Spectrometer (TOMS) e Ozone Monitoring Instrument (OMI), disponibilizados pela National Aeronautics and Space Administration (NASA), e analisados através de médias, valores de anomalias e diagramas hovmoller. Foi constatado que eventos La Niña provocam, predominantemente, aumento na concentração de ozônio sobre o NEB. Nos períodos de atuação desse modo de variabilidade a desaceleração da Circulação Brewer-Dobson (CBD) é causada pela intensificação dos ventos alísios gerando acúmulo de ozônio na região tropical. Entre as duas modalidades, fenômenos Modoki apresentam esse efeito mais intensificado. O maior $(+6,5)$ e menor $(-3,0)$ valor médio mensal de anomalia obtidos ocorreram durante sua atuação, registrados na região norte do NEB em julho e setembro, respectivamente.
\end{abstract}

Palavras-chave: ozônio total, estratosfera, nordeste brasileiro.

\section{Impact of ENSO on Variability of the Total Ozone Column Over the Northeast Region of Brazil - Part 2: La Niña Canonical and Modoki}

\begin{abstract}
This study presents a second stage of the spatio-temporal assessment of long-term trends in ozone concentrations in Northeast Brazil (NEB) under the influence of the El Niño - South Oscillation (ENSO) with emphasis on the La Niña phase (Canonical and Modoki). The objective is to analyze the annual variation and the effects of this phenomenon in the Total Ozone Column (TOC) over the NEB, between 1997 and 2018. The data come from remote sensing, generated by the Total Ozone Mapping Spectrometer (TOMS) and Ozone Monitoring Instrument (OMI) sensors, made available by National Aeronautics and Space Administration (NASA), and analyzed using mean, anomaly values and hovmoller diagrams. It was found that La Niña events cause, predominantly, an increase in the concentration of ozone over the NEB. During periods of action of this mode of variability, the deceleration of Brewer-Dobson circulation (CBD) is caused by the intensification of trade winds, generating ozone accumulation in the tropical region. Between the two modalities, Modoki show this effect more intensified. The highest (+6.5) and lowest (-3.0) average monthly anomaly values obtained occurred during his performance, recorded in the northern region of NEB in July and September, respectively.
\end{abstract}

Keywords: total ozone, stratosphere, northeastern Brazil.

\section{Introdução}

O ozônio se forma na atmosfera da Terra, majoritariamente, através da fotodissociação de oxigênio mole- cular, processo que ocorre predominantemente na estratosfera tropical (Solomon, 1999). Os processos de destruição e transporte determinam a distribuição e concentração

Autor de correspondência: Daniel Milano Costa de Lima E-mail do autor: daniel-milano@hotmail.com 
de ozônio estratosférico ao redor do globo. Enquanto a concentração de ozônio na estratosfera superior (35$50 \mathrm{~km}$ ) é determinada principalmente através de processos fotoquímicos de criação e destruição, na baixa e média estratosfera (abaixo de $30 \mathrm{~km}$ ) é predominantemente definida pelo transporte de massas de ar (Fioletov, 2008; Dias Nunes et al., 2020). As variações dos valores médios da Coluna Total de Ozônio (CTO) ocorrem devido a produção, destruição e transporte de ozônio na estratosfera, e é uma das principais causas das mudanças observadas na circulação atmosférica do Hemisfério Sul (HS) (Salby, 2008; Chehade et al., 2014).

O transporte desse gás na atmosfera dá-se principalmente através da Circulação Brewer-Dobson (CBD) na média e baixa estratosfera, regiões onde sofre reações químicas e fotoquímicas. Esta circulação move parcelas de ar dos trópicos em direção a região polar, acumulando o ozônio, principalmente durante o inverno/primavera de ambos hemisférios, nos extratrópicos e altas latitudes. Quando essa circulação se intensifica a concentração de ozônio aumenta na estratosfera inferior, quando abranda, os valores médios da CTO diminuem ou permanecem constantes, dependendo de seu fluxo na atmosfera (Roscoe, 2006; Weber et al., 2011; Abalos et al., 2015).

O ENOS é um fenômeno caracterizado por possuir componente oceânica (El Niño) e atmosférica (oscilação sul) acopladas, ocorre sobre a região tropical do Oceano Pacífico e é um dos principais moduladores da variabilidade atmosférica interanual (Oliveira e Satyamurty, 1998; Ambrizzi, 2003). Possui duas fases, El Niño e La Niña, e pode atuar em duas modalidades, Canônica ou Modoki (Takahashi, 2011). Maiores definições quanto aos parâmetros para definição de ocorrência, da fase e da modalidade dos fenômenos ENOS foram descritos em Allan (1996), Takahashi (2011) e Philander (1990).

A variação anual é considerada o modo de variabilidade dominante para o ozônio em todo globo terrestre e apresenta uma assinatura meridional com máximos nos extratrópicos, enquanto as variações semestrais e subsazonais são mais aparentes na região equatorial, com tendência de diminuição da magnitude em direção aos pólos (Ganguly e Iyer, 2014; Abalos et al., 2015).

Os eventos ENOS estão ligados às variações dos valores da média zonal de ozônio na estratosfera inferior tropical, em especial nas regiões sobre o Pacífico, através de flutuações na ressurgência tropical associada a CBD (Weber et al., 2011; Ganguly e Iyer, 2014; Xie et al., 2014). Durante a fase El Niño desse fenômeno, o enfraquecimento dos ventos alísios ocasionado por esse fenômeno favorece a aceleração da CBD acarretando maior transporte de ozônio estratosférico da região tropical para a região extratropical (Reed et al., 1961), acumulando-o nessa região (Ganguly e Iyer, 2014). Segundo Reed et al. (1961), o oposto ocorre na fase La Niña do ENOS, resultando em acumulo de ozônio na região equatorial (Zubiaurre e Calvo, 2012).
Estudos de alterações à longo prazo das concentrações de ozônio são abordados em diversos estudos sobre modos de variabilidade climáticos no Hemisfério Sul. Segundo Thohir et al. (2018), a variabilidade do ozônio também é modulada por oscilações sazonais e com durações próximas a dois anos, como a Oscilação QuaseBienal (OQB).

As diferentes fases do ENOS, que determina características na circulação da região extratropical (Reed, 1961; Xie et al., 2012), provocam potencial influência na modulação da geração e propagação de ondas planetárias, como as ondas de Rossby, algo claramente observado nos meses de inverno de ambos os hemisférios (Trenberth et al., 2002). Consoante a Ganguly e Iyer (2014), o ENOS gera anomalias opostas entre a estratosfera e troposfera quanto às variáveis temperatura do ar e concentrações de ozônio.

Entretanto, até o momento, tem-se dado pouca atenção à variabilidade do ozônio na região tropical, principalmente sobre a América do Sul, provocada por fenômenos ENOS (Xie et al., 2012; Lin e Qian, 2019). Durante a primeira etapa desta pesquisa (Lima et al., 2020) foram apresentadas as características espaço-temporais do impacto causado pelo fenômeno ENOS em sua fase El Niño, Canônico e Modoki, na CTO sobre a região do NEB para o período compreendido entre 1997 e 2018. As análises das anomalias da CTO mostraram que o El Niño Modoki tem maior impacto nos valores médios quando comparado à fase Canônica ou períodos sem ocorrência de ENOS. Assim, considerando a influência deste modo de variabilidade climática na distribuição vertical de ozônio em latitudes tropicais e extratropicais, este trabalho tem como objetivo avaliar o impacto de eventos ENOS, com foco na fase La Niña Canônica e Modoki, no comportamento e variabilidade espaço-temporal da CTO sobre a região do NEB.

\section{Materiais e Métodos}

Para quantificar as alterações da CTO na região do NEB foram utilizados dados de sensoriamento remoto de dois dispositivos acoplados a satélites, os sensores Total Ozone Mapping Spectrometer (TOMS) e Ozone Monitoring Instrument (OMI), para o período compreendido entre os anos de 1997 e 2018. Os dados obtidos do sensor TOMS abrange o período de 1997 a 2004, já os dados do sensor OMI, substituto do TOMS (descontinuado em 2005), contempla os anos de 2005 a 2018. Em ambos os sensores a resolução espacial é de $1^{\circ} \times 1^{\circ} \mathrm{em}$ coordenadas geográficas e as concentrações de ozônio na atmosfera são aferidas através de análise do espectro de radiação Ultravioleta (UV) utilizando a técnica Backscatter Ultraviolet (BUV), entregando valores de CTO e perfil em resolução temporal diária. A técnica BUV obtém dados através do mapeamento da radiação UV emitida pelo Sol nas faixas 
espectrais entre $0,270 \mathrm{~nm}$ a $0,314 \mathrm{~nm}$ e $0,306 \mathrm{~nm}$ a $0,380 \mathrm{~nm}$ que é retroespalhada pela atmosfera terrestre em direção ao sensor a bordo do satélite (Bhartia e Wellemeyer, 2002; Herman et al., 2002; McPeters et al., 1998; Wellemeyer et al., 1997; Wellemeyer et al., 2004). Para abranger toda área do NEB foram obtidos dados de 142 quadrantes de mesma dimensão espacial que as resoluções dos sensores TOMS e OMI.

Foram verificadas falhas diárias nas séries de dados (15,8\% dos dados obtidos) e preenchidas utilizando interpolação, entre os dias anteriores e posteriores às mesmas, utilizando a técnica matemática "spline" (Lopes, 2018). Foi avaliada a variabilidade espaço-temporal da CTO nos 142 quadrantes obtidos através de análise de agrupamento (cluster), agregados em cinco diferentes grupos. A quantidade de grupos selecionada é uma característica do método não hierárquico escolhido para o agrupamento (método k-means). Maiores detalhes quanto às metodologias aplicadas podem ser obtidos em Wilks (2011) e Lima et al. (2020).

Assim como na primeira parte deste estudo, onde foi analisada a influência do modo de variabilidade ENOS na CTO com foco apenas na fase El Niño (Canônico e Modoki), nesta segunda etapa é avaliado o modo de variabilidade ENOS na fase La Niña, Canônico e Modoki. Para identificação dos meses sobre influência desse fenômeno foram utilizados os índices The Oceanic Niño Index (ONI), disponibilizado pela NOAA (National Oceanic and Atmospheric Administration), e El Niño Modoki Index (EMI), da JAMSTEC (Japan Agency for Marine-Earth Science and Technology). Nestes índices, valores menores ou iguais à $-0,5$ representam ocorrência de La Niña (Canônica/Modoki), e maiores ou iguais à 0,5 , ocorrência de El Niño (Canônica/Modoki). Os valores no intervalo entre $-0,5$ e 0,5 são considerados períodos neutros (sem atuação de ENOS) (NCEP, 2018). Neste trabalho, para os períodos com e sem ocorrência do fenômeno La Niña (Canônico e Modoki) foram calculadas médias mensais e anomalias da CTO. As listas de meses com ocorrências destes fenômenos estão disponíveis nos sites da NOAA e JAMSTEC. O período estudado abrange 10 eventos canônicos (1998-2001, 2005-06, 2007-08, 2008-09, 2010-12, 2016, 2017-18) e 5 eventos Modoki (1998-99, 2000-01, 2008-09, 2010-11 e 2016-17).

A partir das séries de dados diários de CTO obtidos para cada quadrante foram geradas médias mensais para todos os anos estudados. Essas médias foram associadas aos respectivos valores mensais dos índices ONI e EMI sendo identificado como período de La Niña na ocorrência de índices menores ou iguais a $-0,5$ e sem ocorrência de ENOS na ocorrência de índices entre -0,5 e 0,5. Para obtenção das anomalias, os valores médios obtidos no processo apresentado anteriormente são subtraídos das médias mensais para todo período estudado, cálculos realizados para cada quadrante da área do estudo.
Foram analisadas as médias e anomalias mensais e sazonais para os trimestres Dezembro-Janeiro-Fevereiro (DJF) e Junho-Julho-Agosto (JJA), para toda a área do NEB. Para setorizar as diferentes regiões do NEB por características do comportamento médio da CTO foi utilizada análise de agrupamento, resultando em grupos de quadrantes que posteriormente foram analisados através de médias e anomalias permitindo caracterizar individualmente cada uma das subregiões. Utilizando os dados gerados nos processos apresentados anteriormente, para as duas modalidades de La Niña foram gerados diagramas Hovmöller da variação interanual média da CTO pela latitude e histogramas contendo análise dos valores de anomalia de cada quadrante com o objetivo de avaliar as intensidades das anomalias. Destaca-se novamente que maiores detalhes da metodologia apresentada podem ser encontrados em Lima et al. (2020).

\section{Resultados e Discussões}

A Fig. 1 apresenta as médias de CTO e anomalias médias para os períodos com ocorrência de eventos de La Niña nas fases Canônica e Modoki entre 1997 e 2018. Os quadros superiores representam toda a área do NEB, expondo, alternadamente, os valores médios e os valores das anomalias. Características das fases Canônica são exibidas na Fig. 1a e Modoki na Fig. 1b. Com relação aos campos médios da CTO e sua variabilidade, semelhante ao encontrado por Xie et al. (2014a; 2014b), em ambas as fases, há homogeneidade de valores na área do NEB, apresentando números entre 258,0 e 266,0 UD, uma variação em torno de $3,0 \%$. Os menores valores estão sobre a região sudoeste do NEB abrangendo o oeste da Bahia, o sul do Maranhão e do Piauí. Os maiores estão compreendidos entre o centro da área do NEB e a costa. Para La Niña Canônico, os maiores valores médios se apresentam durante o período agosto-novembro, que por sua vez também possuem as maiores anomalias encontradas, com destaque para as latitudes de $8^{\circ}$ e acima (Zhang et al., 2015).

A variabilidade mensal da CTO na região do NEB tem seus menores valores entre os meses de abril e junho, com valores próximos a $242 \mathrm{UD}$, e máximos nos meses entre julho a novembro, com valores chegando próximos a 285 UD. Nos diagramas Hovmöller, para ambas modalidades, fica evidente o aumento da concentração da CTO nos meses entre julho a novembro, como mostrado em Shangguan et al. (2019). Neste período também é observada uma assimetria da CTO ao longo das latitudes, mostrando que a área de aumento dos valores médios da CTO tende a ser menor na faixa latitudinal entre $12^{\circ} \mathrm{S}$ e $15^{\circ} \mathrm{S}$ (Fig. 1).

No período de ocorrência dos eventos Canônicos (Fig. 1a) observa-se anomalias positivas em toda a extensão da área estudada, valores entre 0,2 e 1,2, implicando 


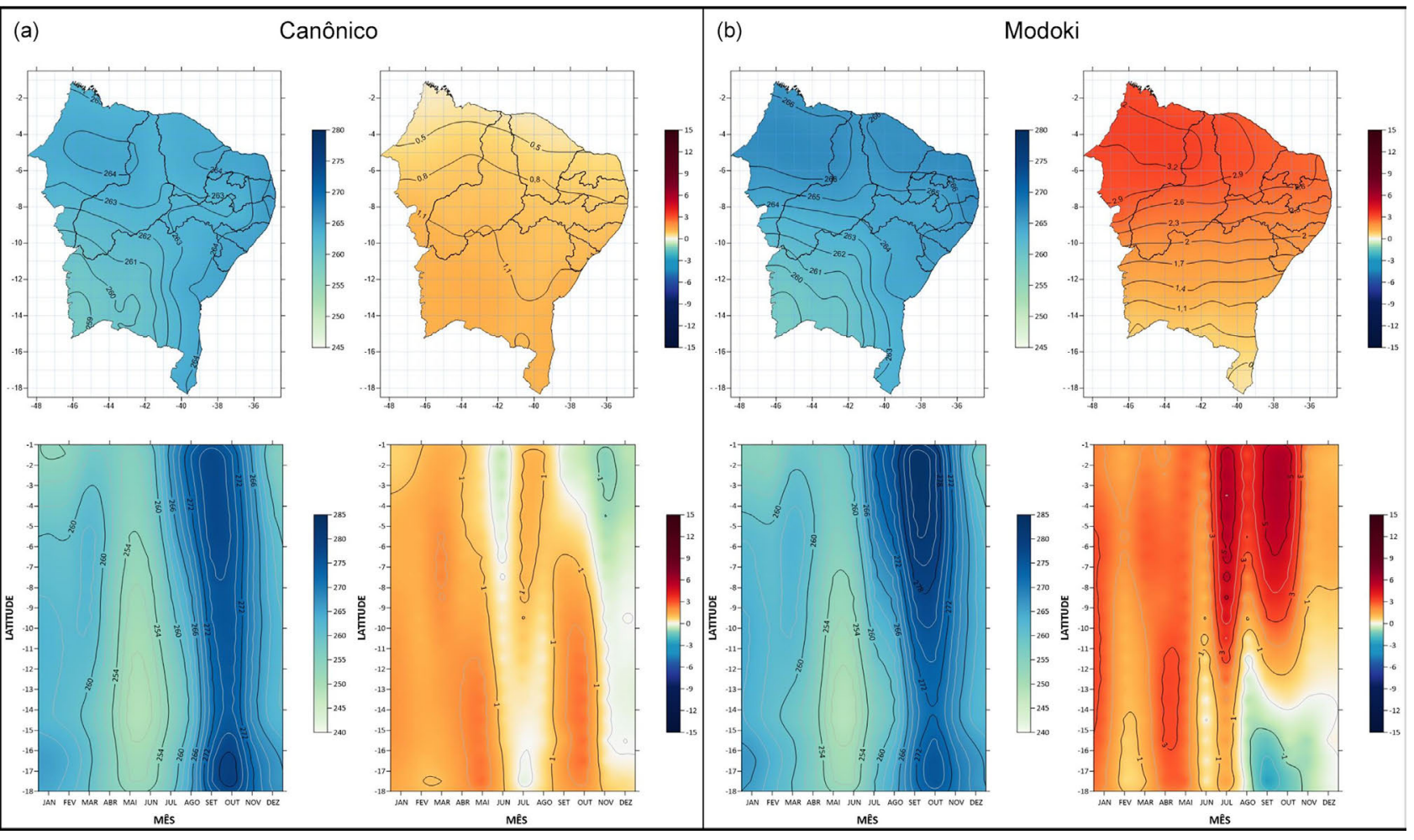

Figura 1 - No quadro (a), La Niña Canônico, e (b), La Niña Modoki. Na primeira coluna de cada quadro, dados de valores médios de CTO para o período entre 1997 e 2018. Na segunda, dados relativos a anomalia média da CTO para o mesmo período. Na linha superior, gráficos de área para todo NEB expondo valores médios para o período entre 1997 e 2018. Na inferior, diagrama Hovmöller com média mensal dos valores dos quadrantes de mesma latitude.

que estes eventos tendem a, em visão generalizada, aumentar os valores médios da CTO sobre o NEB. Os menores valores se apresentam no extremo norte da região e aumentam à medida que avançamos para maiores latitudes. Anomalias positivas predominam em todas as latitudes para quase todos os meses do ano, como mostra a Fig. 1a (diagrama hovmoller apresentado no painel inferior direito). As poucas médias de anomalias negativas registradas estão presentes nos últimos meses do ano em quase todas latitudes, mais intensas nas proximidades do equador semelhante ao encontrado por Xie et al. (2014a; 2014b), Zhang et al. (2015) e Shangguan et al. (2019).

Para eventos Modoki, assim como os Canônicos, os valores de anomalia de todo período sob sua influência são positivos (Fig. 1b, superior direita). Os dados resultantes variam seus valores entre 0,4 e 3,3, superior aos limites da média obtida para a fase canônica. Isso mostra que, numa visão generalizada, o La Niña Modoki provoca influência semelhante ao La Niña Canônico, aumentando os valores de CTO sobre o NEB, no entanto de forma mais acentuada e com maior influência em latitudes menores (Xie et al., 2014a). As anomalias perdem intensidade à medida que nos afastamos dessa região. Nenhuma latitude menor que $11^{\circ} \mathrm{S}$, segundo o diagrama Hovmöller (Fig. 1b, inferior direita), apresenta anomalias negativas entre as médias latitudinais mensais. Em latitudes maiores que $11^{\circ} \mathrm{S}$ as anomalias positivas são mais intensas entre junho e novembro. Anomalias negativas predominam nas maiores latitudes do NEB durante todo o segundo semestre e têm seus maiores valores em setembro. Durante ocorrência de ENOS em fase La Niña (Canônico e Modoki) há predominância do gradiente de temperatura negativo, ou seja, o gradiente aponta da região equatorial em direção a região polar (Reed, 1961), o que favorece a desaceleração da CBD (que é balanceada na região tropical pela circulação meridional induzida pela QBO). As anomalias negativas do vento zonal equatorial produzem esta desaceleração e como consequência acarretam diminuição dos movimentos ascendentes na região equatorial, levando ao acúmulo de ozônio nesta mesma região devido a redução do transporte para regiões de altas latitudes. Sendo assim, há o predomínio de anomalias positivas da CTO nos trópicos e negativas nos extratrópicos (Reid e Cage, 1985; Zhang et al., 2015).

O painel apresentado na Fig. 2 indica um padrão bastante similar de médias e anomalias entre os trimestres DJF e JJA para análises da ação de La Niña Canônico (linha superior) e Modoki (linha inferior). As médias referentes ao trimestre DJF apresenta maiores valores na região sudeste da área de estudo (costa leste do NEB, em maiores latitudes). Neste trimestre percebe-se disparidades ao se comparar as duas modalidades de La Niña quando 
analisadas as anomalias. Nota-se um padrão de maiores valores quando existe a ocorrência de La Niña Modoki com destaque para latitudes mais próximas à região equatorial. Como mostrado por Xie et al. $(2012$; 2014) em fase oposta (El Niño), nos períodos de La Niña (Canônico e Modoki), a desaceleração da CBD é causada pela intensificação dos ventos alísios, o que gera um acúmulo de ozônio na região tropical, mostrada pelos valores anomalamente positivos nesta região como visto na Fig. 2.

O trimestre DJF é representado no lado esquerdo do painel e o trimestre JJA é representado no lado direito do painel, onde cada painel apresenta os valores médios de cada trimestre (tons crescentes de amarelo para azul) e as anomalias positivas e negativas (vermelho a azul escuro).

O trimestre JJA (Fig. 2b) apresenta valores médios, semelhante ao já destacado no trimestre DJF, sem muita variação entre as duas modalidades de La Niña estudadas. $\mathrm{O}$ destaque novamente ocorre quando se comparam os valores das anomalias provocadas por eventos Canônicos e Modoki. Diferente do apresentado no trimestre DJF, o trimestre JJA possui discrepâncias entre as duas modalidades, semelhante ao encontrado em Lin e Qian (2019). Nesse trimestre, durante a ocorrência do modo canônico notam-se anomalias positivas em toda a área de estudo. Entretanto, durante a ocorrência da fase Modoki, grandes valores de anomalias positivas são encontrados, sobretudo na região mais próxima ao equador, e diminuem em direção a maiores latitudes, efeito provocado pela desaceleração da CBD, o que favorece as anomalias positivas de CTO na região equatorial (Xie et al., 2012; 2014).
Para a fase Canônica, conforme apresentado na Fig. 3a, a maior parte das anomalias médias mensais obtidas dos quadrantes estudados são positivas, sendo $80 \%$ dos registros com essa característica, com valores variando entre 0 e 2,8. O intervalo de amplitude 0,5 com o maior montante de registros está entre 1,0 e 1,5, com 406 registros de valores de quadrantes $(29,6 \%$ das anomalias computadas). $34,4 \%$ das anomalias positivas estão entre $1,5 \mathrm{e}$ 3,0 , e $36 \%$ entre 0 e 1,0. Para as anomalias negativas foram registrados 333 valores, $20 \%$ dos casos registrados, todos com intensidade variando entre 0 e $-1,8$.

Semelhante a fase Canônica, em períodos de influência de eventos tipo Modoki foram registradas maiores quantidade de anomalias médias mensais positivas ao se analisar todos os quadrantes da área de estudo. De todos os registros, 1582 valores de anomalias médias mensais são positivas ( $93 \%$ do total de registros), ocupando mais áreas com valores deste sinal quando comparada à fase Canônica. O histograma revela que os valores positivos se distribuem de 0 a 6,5 (Fig. 3b). A faixa predominante dos valores se apresenta entre 0 e $3,5(82,9 \%)$ seguido de 3,5-6,5 (17,1\% da ocorrência dos dados). Essas características revelam que a La Niña, Canônica e Modoki, atua, predominantemente, aumentando os valores médios da CTO sobre o NEB, no entanto, com maior intensidade quando comparado com os efeitos identificados durante a ocorrência de El Niño Canônico ou Modoki (Lima et al., 2020). Anomalias negativas apresentam valores entre 0 e $-3,0$, com $87,7 \%$, dos valores negativos, entre 0 e $-1,0$.

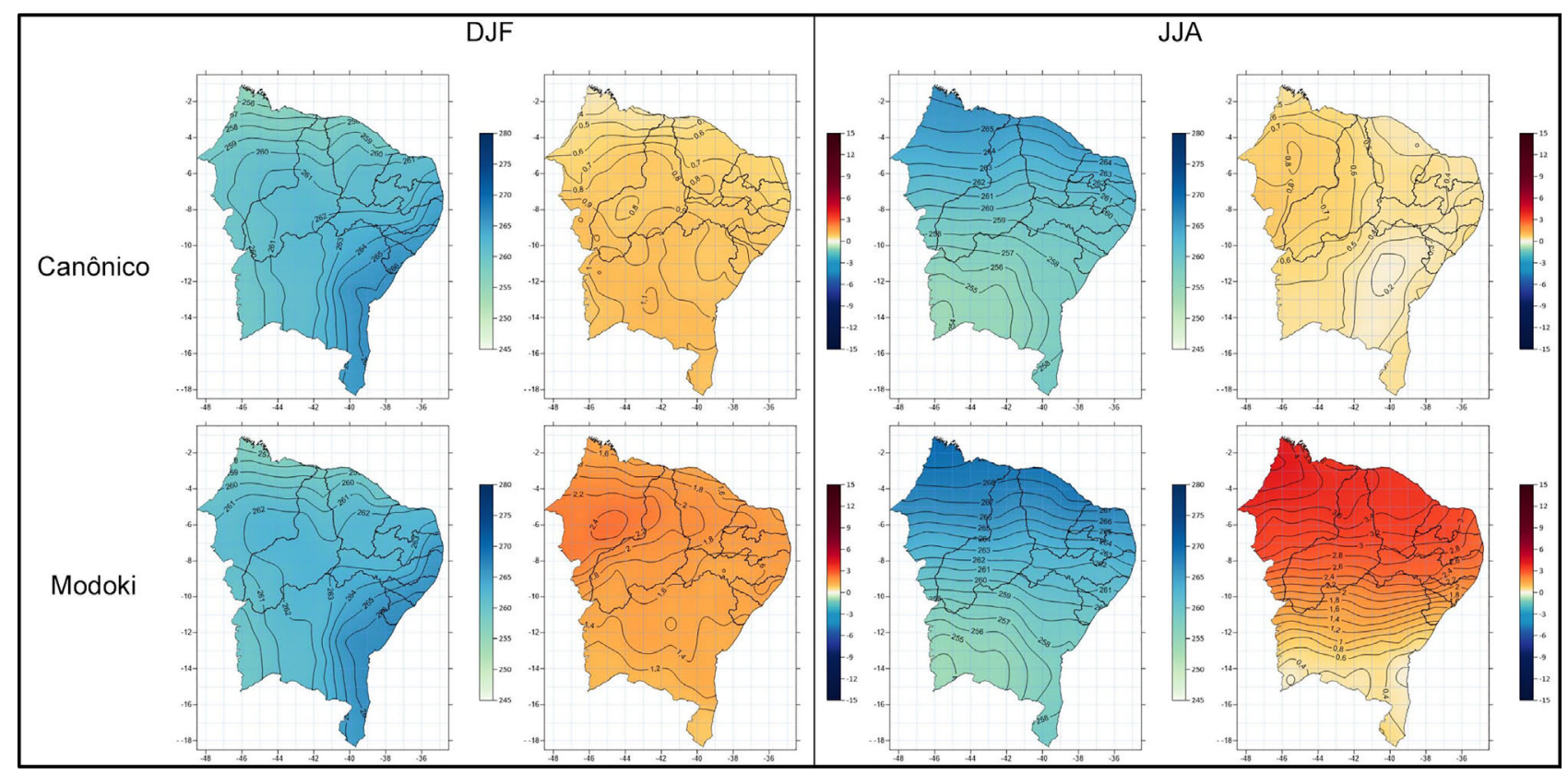

Figura 2 - No painel à esquerda, valores médios sazonais e anomalias médias da CTO sobre a área do NEB durante ação de fenômenos La Niña no trimestre DJF. No painel à direita, durante o trimestre JJA. Na primeira coluna de cada quadro, dados de valores médios de CTO. Na segunda, dados relativos à anomalia média da CTO. Nas linhas, gráficos de área para todo NEB expondo valores médios para o período entre 1997 e 2018. 

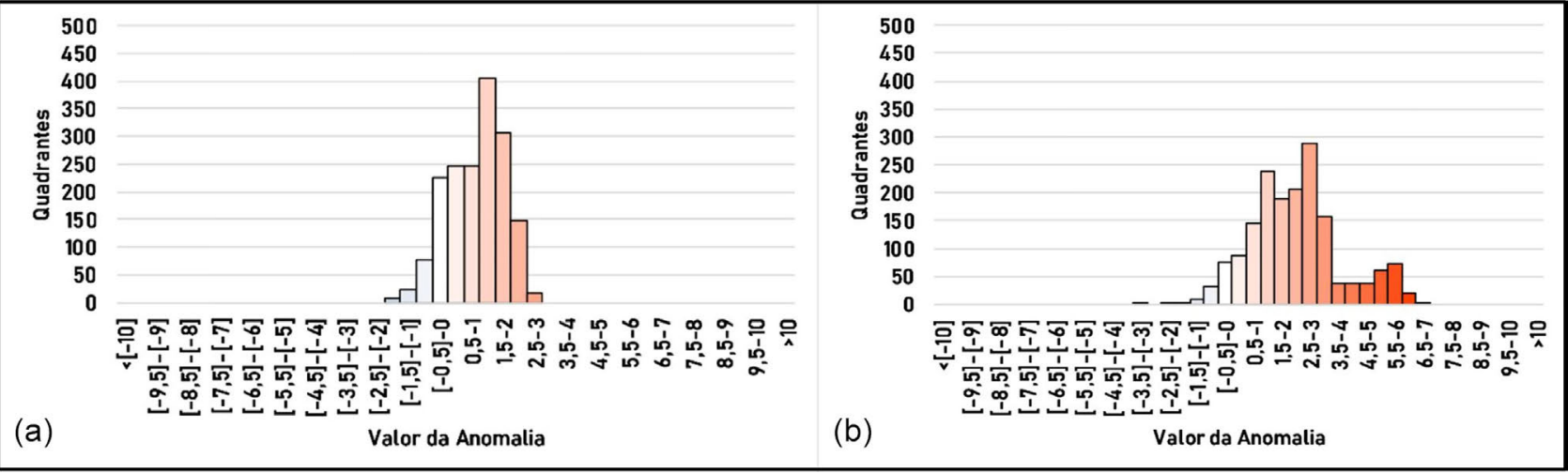

Figura 3 - Histograma dos valores médios mensais das anomalias de todos os quadrantes. Os períodos analisados são: sob a influência de La Niña Canônico (a) e sob influência de La Niña - Modoki (b).

A análise comparativa da variabilidade das anomalias mensais médias de CTO para toda a área do NEB sob a influência das fases La Niña Canônico e Modoki revela diferenças sazonais consideráveis principalmente nos meses entre julho e novembro para a análise média dos eventos ocorridos entre 1997 a 2018 (Fig. 4). Na fase Modoki os valores anômalos são, predominantemente, positivos e apresentaram em todos os meses valores maiores do que os valores médios mensais da CTO durante a influência de La Niña Canônico, o que ratifica o encontrado nas análises anteriores (Fig. 1). Estas anomalias positivas estão associadas ao enfraquecimento/desaceleração da $\mathrm{CBD}$, que ocorre na fase La Niña de ENOS, como citado por Reed (1961); Reid e Cage (1985), o que modula a CTO na região tropical, como mostrado por Xie et al. (2014a; 2014b); Zhang et al. (2015); Shangguan et al. (2019). Destaca-se ainda a ocorrência de uma maior variação em torno da média durante estes eventos, com destaque para os meses entre julho-outubro. Diferente do La Niña - Canônico que apresenta médias mensais para a área analisada com valores negativos nos meses de junho, novembro e dezembro, o fenômeno Modoki apresenta médias positivas para todos os meses, todas superiores às médias canônicas.

A Fig. 4 apresenta a média, máximos e mínimos valores médios mensais de CTO encontrados sobre toda região NEB quando sob efeito de eventos La Niña de ambas modalidades. Na comparação entre Canônico e Modoki nota-se um padrão divergente principalmente nos valores mínimos apresentados. Para La Niña Canônicos, percebe-se a existência, em ao menos um dos quadrantes dentro da região, de oito meses com anomalias negativas, enquanto para Modoki apenas seis. Destaque para o mês de maio quando sobre a ocorrência de La Niña Canônica no qual constatou-se valor mínimo encontrado $(-0,4)$. Para La Niña Modoki o mesmo ocorre, com valor de registro 2,2. Em eventos Modoki, de janeiro a maio toda a área do NEB apresenta apenas valores mínimos positivos, entre 0,5 e 2,6 .
Os meses entre janeiro e abril apresentam, em ambas as modalidades, apenas valores máximos e mínimos positivos, entre 0,4 e 2,4. Dentre esses meses, março apresenta a maior média para eventos canônicos $(1,8)$ e julho $(4,6)$ para eventos na modalidade Modoki, semelhante ao encontrado por Manatsa e Mukwada (2017), que avaliaram o comportamento da CTO na região tropical no período compreendido entre Dezembro e Março de 1986 a 2016. Quando analisados os valores médios destaca-se a ocorrência de maiores registros durante eventos La Niña Modoki quando comparado aos Canônicos. Esse fato, juntamente com os dados apresentados na Fig. 4, indica a existência de maiores valores de CTO quando a atmosfera tropical está sobre influência daquele modo de variabilidade climática. Por sua vez, como apresentado por Xie et al. (2014a), Zhang et al. (2015) e Shangguan et al. (2019), isso pode ser um indicativo de como a estratosfera, e consequentemente a circulação CBD, pode ser afetada durante esses eventos.

\section{Conclusões}

O presente trabalho teve como principal objetivo investigar as variações interanuais e mensais da CTO sobre a região do NEB sob efeito de influência do modo de variabilidade climático ENOS - La Niña Canônico e Modoki no período compreendido entre 1997 e 2018.

No estudo por quadrantes das anomalias médias mensais para períodos de atuação de La Niña Canônico, $80 \%$ dos valores obtidos são de anomalias positivas e $20 \%$ de anomalias negativas. Durante eventos Modoki, 93\% dos dados exibem anomalias positivas e $7 \%$ anomalias negativas. Os valores médios de CTO para o trimestre DJF apresentam máximos na região sudeste do NEB, costa leste da região em maiores latitudes para ambas modalidades de La Niña. Quantos às médias de anomalia, também ambas modalidades de La Niña apresentam valores positivos, no entanto, eventos Modoki apresentam maiores valores médios quando comparado à fenômenos canôni- 


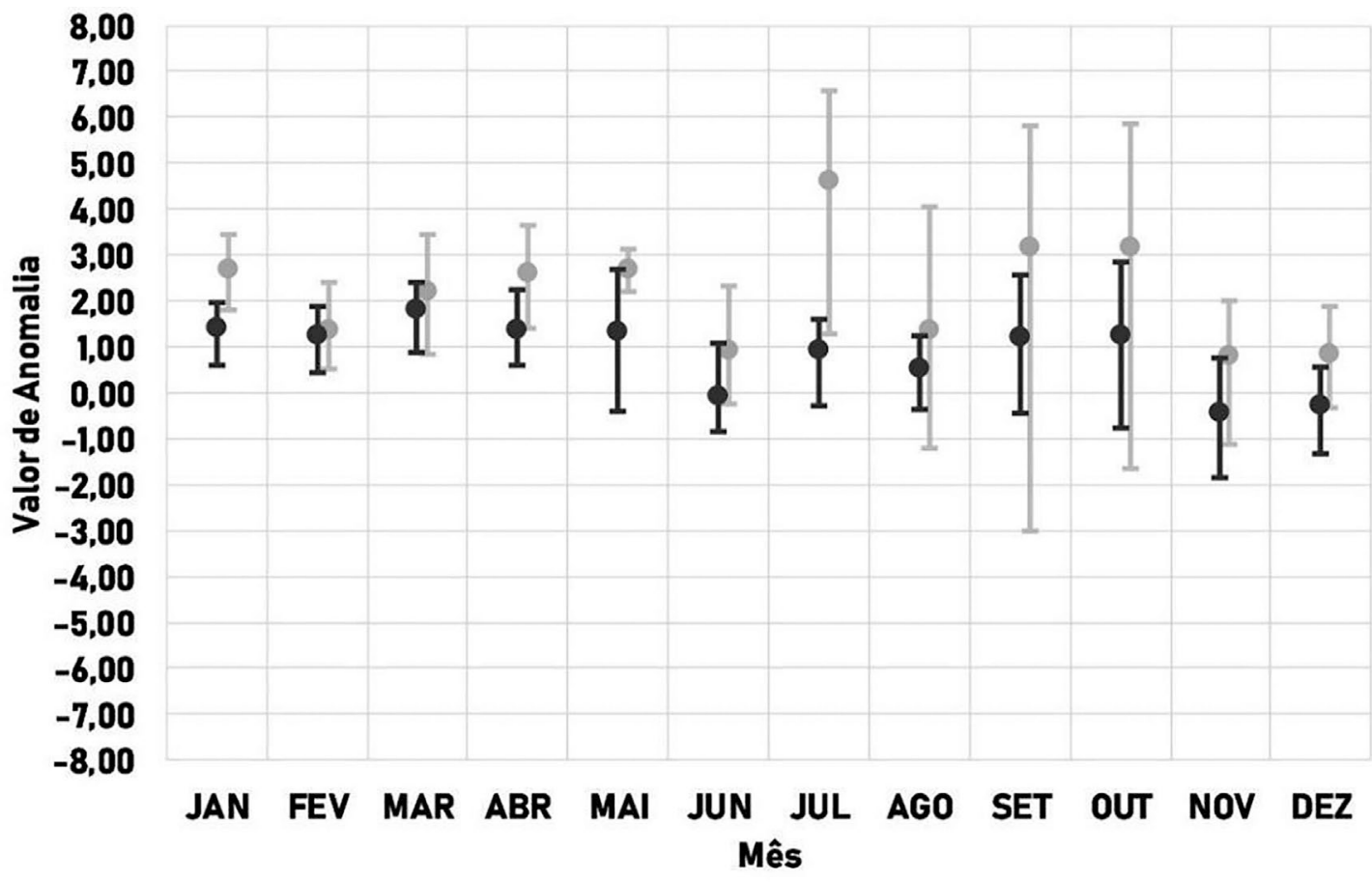

LA NIÑA CANÔNICO

LA NIÑA MODOKI

\begin{tabular}{|c|c|c|c|c|c|c|c|c|c|c|c|c|c|}
\hline \multirow{2}{*}{\multicolumn{2}{|c|}{ LEGENDAS: }} & \multicolumn{12}{|c|}{ MÊS } \\
\hline & & JAN & FEV & MAR & ABR & MAI & JUN & JUL & $A G O$ & SET & OUT & NOV & DEZ \\
\hline \multirow{2}{*}{$\begin{array}{l}\text { VALOR MÁXIMO } \\
\text { REGISTRADO }\end{array}$} & CANÔNICO & 1,95 & 1,89 & 2,41 & 2,26 & 2,69 & 1,10 & 1,60 & 1,22 & 2,58 & 2,84 & 0,75 & 0,55 \\
\hline & MODOKI & 3,47 & 2,40 & 3,46 & 3,66 & 3,13 & 2,35 & 6,57 & 4,05 & 5,81 & 5,87 & 2,01 & 1,89 \\
\hline \multirow{2}{*}{$\begin{array}{l}\text { VALOR MINIMO } \\
\text { REGISTRADO }\end{array}$} & CANÔNICO & 0,62 & 0,45 & 0,88 & 0,59 & $-0,40$ & $-0,86$ & $-0,29$ & $-0,37$ & $-0,43$ & $-0,77$ & $-1,84$ & $-1,32$ \\
\hline & MODOKI & 1,82 & 0,51 & 0,85 & 1,42 & 2,22 & $-0,23$ & 1,27 & $-1,22$ & $-3,02$ & $-1,63$ & $-1,11$ & $-0,31$ \\
\hline \multirow{2}{*}{$\begin{array}{l}\text { MÉDIA PARA } \\
\text { TODO NEB }\end{array}$} & CANÔNICO & 1,45 & 1,27 & 1,86 & 1,40 & 1,35 & $-0,02$ & 0,95 & 0,57 & 1,22 & 1,28 & $-0,41$ & $-0,26$ \\
\hline & MODOKI & 2,72 & 1,40 & 2,26 & 2,66 & 2,73 & 0,97 & 4,66 & 1,42 & 3,19 & 3,20 & 0,84 & 0,88 \\
\hline
\end{tabular}

Figura 4 - Comparativo de valores mensais médios, máximos e mínimos de CTO para períodos sob influência de La Niña Canônico e Modoki. No gráfico superior, círculos preenchidos representam valores médios mensais e barras indicam valores máximos e mínimos registrados para os respectivos meses.

cos, apresentando números expressivamente maiores nas latitudes mais próximas à região equatorial.

$\mathrm{O}$ trimestre JJA apresenta valores médios próximos aos do trimestre DJF, sem muita variação entre as duas modalidades de La Niña. Para médias de anomalias, discrepante ao trimestre DJF, JJA possui características diferenciadas entre as modalidades. Durante a ocorrência do modo canônico percebe-se anomalias positivas em toda a área de estudo, durante eventos Modoki valores ainda maiores são encontrados, sobretudo na região mais próxima ao equador, diminuindo em direção à maiores latitudes.

O aumento na concentração de ozônio ocorre pois durante ocorrência de eventos La Niña (Canônico e Modoki) há predominância do gradiente de temperatura nega- tivo, ou seja, o gradiente aponta da região equatorial em direção a região polar (Reed, 1961) desencadeando um aumento dos ventos de leste, o que favorece a desaceleração da $\mathrm{CBD}$ (que é balanceada na região tropical pela circulação meridional induzida pela QBO). As anomalias negativas do vento zonal equatorial produzem esta desaceleração e como consequência acarretam diminuição dos movimentos ascendentes na região equatorial, o que leva ao acúmulo de ozônio nesta mesma região devido a redução do transporte para regiões de altas latitudes (Reid e Cage, 1985; Zhang et al., 2015). As diferenças de intensidades das anomalias entre as duas modalidades de La Niña, sendo maiores para fenômenos Modoki, concordam com o apresentado por Xie et al. (2014a); Zhang et al. (2015) e Shangguan et al. (2019), os quais apontam que 
pode ser um indicativo de como a estratosfera, e consequentemente a circulação $\mathrm{CBD}$, pode ser afetada durante esse evento.

Esse resultado tem grande valor pois revela que em períodos sob atuação de fenômenos La Niña, Canônico e Modoki, há tendência de aumento da concentração de ozônio na região do NEB, o que consequentemente diminui o saldo de radiação UV que atinge a superfície terrestre, assim resguardando as diversas formas de vida presentes nesta região sensíveis a este tipo de radiação.

A quantidade de eventos Canônico e Modoki contidas no período analisado pode ter influência no resultado obtido, tornando necessário, futuramente, uma análise mais ampla abrangendo um maior período de estudo. Os resultados obtidos incentivam futuras pesquisas envolvendo outros modos de variabilidade atmosférica que atuam sobre a região com objetivo de compreender se estes também influenciam nas concentrações de CTO.

\section{Referências}

ABALOS, M.; LEGRAS, B.; PLOEGER, F.; RANDEL, W.J. Evaluating the advective Brewer-Dobson circulation in three reanalyses for the period 1979-2012. Journal of Geophysical Research Atmospheres, v. 120, n. 15, p. 7534-7554, 2015.

BHARTIA, P.K.; WELLEMEYER, C. OMI Algorithm Theoretical Basis Document: TOMS-V8 Total O3 Algorithm. NASA Goddard Space Flight Cent, v. 2, p. 15-31, 2002. Disponível em: https://eospso.gsfc.nasa.gov/sites/default/ files/atbd/ATBD-OMI-02.pdf.

CHEHADE, W.; WEBER, M.; BURROWS, J.P. Total ozone trends and variability during 1979-2012 from merged data sets of various satellites. Atmospheric Chemistry and Physics, v. 14, n. 13, p. 7059-7074, 2014.

DIAS NUNES, M.; MARIANO, G.L.; PERES, L.V.; LIMA, D.M.C. Ozônio estratosférico: climatologia global, variabilidade e tendências. In: Glauber Lopes Mariano; André Becker Nunes (Orgs). Meteorologia em Tópicos - Volume 6. Maceió: Clube dos Autores, p. 177-230, 2020.

FIOLETOV, V.E. Ozone climatology, trends, and substances that control ozone. Atmosphere-Ocean, v. 46, n. 1, p. 39-67, 2008.

GANGULY, N.D.; IYER, K.N. Can El Niño induce stratospheretroposphere exchange of ozone? Current Science, v. 106, n. 6, p. 860-866, 2014.

GONZALEZ, P.L.; POLVANI, L.M.; SEAGER, R.; CORREA, G.J. Stratospheric ozone depletion: a key driver of recent precipitation trends in South Eastern South America. Climate Dynamics, v. 42, n. 7-8, p. 1775-1792, 2014.

HERMAN, J.R.; BHARTIA, P.K.; KRUEGER, A.J.; MCPETERS, R.D.; WELLEMEYER, C.G.; SEFTOR, C.J.; JAROSS, G.; SCHLESINGER, B.M.; TORRES, O.; LABOW, G.; BYERLY, W.; TAYLOR, S.L.; SWISSLER, T.; CEBULA, R.P.; GU, X.Y. Meteor-3 Total Ozone Mapping Spectrometer (TOMS) Data Products User's Guide. Washington, D.C.: NASA, 1996.
LIMA, D.M.C; DIAS NUNES, M.; MARIANO, G.L. Impacto do ENOS na variabilidade da Coluna Total de Ozônio sobre a região Nordeste do Brasil - Parte 1: El Niño Canônico e Modoki. Revista Brasileira de Meteorologia v. 35, n. 5, p. 945-953, 2020.

LOPES, Á.P.; COSTA, M.J.S. Comparação entre métodos de aproximação numérica utilizando o programa MATLAB. Revista Margens Interdisciplinar, v. 11, n. 17, p. 14, 2018.

MANATSA, D.; MUKWADA, G. A connection from stratospheric ozone to El Niño-Southern Oscillation. Scientific Reports, v. 7, n. 1, p. 1-10, 2017.

MCPETERS, R.D.; BHARTIA, P.K.; KRUEGER, A.J.; HERMAN, J.R.; WELLEMEYER, C.G.; SEFLOR, G.; JAROSS, C.F.; TORRES, O.; MOY, L.; ABOW, G.; BYERLY, W.; TAYLOR, S.L.; SWISLER, T.; CEBULA, R.P. Earth Probe Total Ozone Mapping Spectrometer (TOMS) Data Products User Guide. Washington, D.C.: NASA, 1998.

REED, R.J.; CAMPBELL, W.J.; RASMUSSEN, L.A.; ROGERS, D.G. Evidence of downward propagating annual wind reversal in the equatorial stratosphere. Journal of Geophysical Research, v. 66, n. 3, p. 813-818, 1961.

REID, G.C.; GAGE, K.S. Inter-annual variations in the height of the tropical tropopause. Journal of Geophysical Research, v. 90, n. D3, p. 5629-5635, 1985.

ROSCOE, H.K. The Brewer-Dobson circulation in the stratosphere and mesosphere-Is there a trend? Advances in Space Research, v. 38, n. 11, p. 2446-2451, 2006.

SALBY, M.L. Involvement of the Brewer-Dobson circulation in changes of stratospheric temperature and ozone. Dynamics of Atmospheres and Oceans, v. 44, n. 3-4, p. 143-164, 2008.

SHANGGUAN, M.; WANG, W.; JIN, S. Variability of temperature and ozone in the upper troposphere and lower stratosphere from multi-satellite observations and reanalysis data. Atmospheric Chemistry and Physics, v. 19, n. 10, p. 6659-6679, 2019.

SOLOMON, S. Stratospheric ozone depletion: a review of concepts and history. Reviews of Geophysics, v. 37, n. 3, p. 275-316, 1999.

TOIHIR, A.M.; PORTAFAIX, T.; SIVAKUMAR, V.; BENCHERIF, H.; PAZMIÑO, A.; BËGUE, N. Variability and trend in ozone over the southern tropics and subtropics. Annales Geophysicae, v. 36, n. 2, p. 381-404, 2018.

WEBER, M.; DIKTY, S.; BURRWS, J. P.; GARNY, H.; DAMERIS, M.; KUBIN, A.; ABALICHIN, J.; LANGAMATZ, U. The Brewer-Dobson circulation and total ozone from seasonal to decadal time scales. Atmospheric Chemistry and Physics, v. 11, n. 21, p. 11221-11235, 2011.

WELLEMEYER, C.G.; TAYLOR, S.L.; SEFTOR, C.J.; MCPETERS, R.D.; BHARTIA, P.K. A correction for the Total Ozone Mapping Spectrometer profile shape errors at high latitude. J. Geophys. Res., v. 102, n. D7, p. 90299038, 1997.

WELLEMEYER, C.G.; BHARTIA, P.K.; MCPETERS, R.D.; TAYLOR, S.L.; AHN, C. A new release of data from the Total Ozone Mapping Spectrometer (TOMS). SPARC Newsletter, v. 22, n. 1, p. 37-38, 2004.

WILKS, D. Statistical Methods in the Atmospheric Sciences, Cambridge: Academic Press, 704 p, 2011. 
XIE, F.; LI, J.; TIAN, W.; ZHANG, J.; SHU, J. The impacts of two types of El Niño on global ozone variations in the last three decades. Advances in Atmospheric Sciences, v. 31, n. 5, p. 1113-1126, 2014a.

XIE, F.; LI, J.; TIAN, W.; ZHANG, J.; SHU, J. The relative impacts of El Niño Modoki, canonical El Niño, and QBO on tropical ozone changes since the 1980s. Environmental Research Letters, v. 9, 064020, 2014b.

ZHANG, J.; TIAN, W.; WANG, Z.; XIE, F.; WANG, F. The influence of ENSO on northern midlatitude ozone during the winter to spring transition. Journal of Climate, v. 28, n. 12, p. 4774-4793, 2015.

ZIEMKE, J.R.; CHANDRA, S.; OMAN, L.D.; BHARTIA, P.K. A new ENSO index derived from satellite measurements of column ozone. Atmospheric Chemistry \& Physics Discussions, v. 10, n. 2, p. 3711-3721, 2010.
ZUBIAURRE, I.; CALVO, N. The El Niño-Southern Oscillation (ENSO) Modoki signal in the stratosphere. Journal of Geophysical Research: Atmospheres, v. 117, D04104, 2012.

\section{Endereços de Internet}

JAMSTEC. http://www.jamstec.go.jp/aplinfo/sintexf/e/elnmo doki/data.html

NOAA. https://origin.cpc.ncep.noaa.gov/products/analysis_moni toring/ensostuff/ONI_v5.php

License information: This is an open-access article distributed under the terms of the Creative Commons Attribution License (type CC-BY), which permits unrestricted use, distribution and reproduction in any medium, provided the original article is properly cited. 